\title{
El estilo norteamericano: su pasado, sus principios
}

\begin{abstract}
STANLEY HOFFMAN es Professor of Government en la Universidad de Harvard; autor de Contemporary Theory in International Relations, The State of War. Su libro más reciente, escrito para el Council on Foreign Relations, Nueva York, es Gulliver's Troubles or The Setling of American Foreign Policy. El presente articulo ha sido reproducido con permiso especial del autor y de Foreign Affairs. (Los derechos pertenecen al Council on Foreign Relations, Inc., Nueva York).
\end{abstract}

Aunque el concepto de un carácter nacional haya resultado de una validez dudosa, el de un estilo nacional contiene mayores promesas. Es un postulado y una interpretación. Intenta establecer orden dentro de una caótica masa de rasgos, al proponer que una nación percibe el mundo, y su lugar en aquél, de un modo nunca exactamente similar al de ninguna otra nación, tal como un individuo nunca enfrenta el mundo exactamente del mismo modo que otro. Es un procedimiento de selección y por ello inevitablemente, uno de exclusión, y es a su vez un procedimiento de distorsión, porque los factores seleccionados son refractados a través del prisma propio del carácter de la nación o del individuo.

Tres aspectos de la singularidad estadounidense son pertinentes al estudio de la politica extêrior: su pasado, sus principios y su pragmatismo. Los tres forman parte de una experiencia nacional cuy'a importancia respecto al mundo exterior es incierta, porque las condiciones en que ésta ha acaecido han sido tan distintas a las condiciones en cualquier otro lugar. Y sin embargo los estadounidenses no han dejado jamás de proyectar hacia su politica exterior las tres facetas de su experiencia. Su componente histórico (lecciones aprendidas y hábitos mentales derivados de la singular situación de su pais); la manera norteamericana de pensar, de percibir mentalmente el mundo, para formar juicios y realizar reformas (una especie de racionalización de la experiencia de Estados Unidos); y el modo de actuar norteamericano, de percibir instrumentalmente al mundo, constituyen las herramientas que han logrado que la experiencia norteamericana sea un éxito.

En este articulo dejaré fuera el pragmatismo estadounidense y me concentraré sobre un aspecto de cada uno de los otros dos rasgos: ¿Cuál ha sido el legado dejado por el pasado de Estados Unidos a la actitud 
Stanley Hoffmann / El estilo norteamericano: su pasado, sus principios

norteamericana hacia la historia? ¿Y cuál es la naturaleza de los principios norteamericanos?

Puede servir de gran entretención para los exploradores aficionados (no existen otros) de siques nacionales el establecer una tipologia de actitudes nacionales hacia la historia, abarcando quizás desde el rousseauniano y a menudo mitico sentido de las naciones nuevas, de que una largamente enterrada edad de oro fue seguida por una larga noche de corrupción, que precedió al reciente despertar de la independencia, hasta el sentido de la historia de las antiguas naciones, como un océano dueño de una larga crónica ya desdibujada de tempestades y momentos de calma. En la mayoria de los casos la historia se ve como una lucha - ya sea una sucesión de embrollos sin significado último u orden discernible, o una lenta y fatigante emancipación, desde las diferentes formas de servidumbre hasta variables grados de esclarecimiento. Para los filósofos y los ideólogos que pretenden encontrar una línea en su crónica, un significado en el enredo, la historia es un magulla do testigo constantemente solicitado a presentarse frente al tribunal; para los agnósticos históricos, la historia es una prenda en llamas que se adhiere a nuestras pieles, un libro de cuentos que no comprueba nada.

La actitud norteamericana hacia la historia se me presenta muy diferente; tenemos la tendencia de considerarla plácidamente y desde cierta distancia. Los norteamericanos saben que están en ella, que su prenda quema, pero no logran percatarse suficientemente de lo: imposible que es quitarse el ropaje o usarlo sin incomodidad. La actitud es de satisfacción, pero esta satisfacción se ve amenazada.

En Estados Unidos, donde el conservantismo es la conservación del progreso, ha existido - por contraste con Europa - una especie de feliz simplicidad que es a la vez una bendición doméstica y un obstáculo para la comprensión, una fuente de energia interna y de empobrecimiento mental y emocional. La respuesta europea ante la crisis y el desafio ha sido infinitamente variada - los europeos están acostumbrados a las altas y bajas, vueltas y revueltas, oportunidades propicias y desfavorables. En consecuencia, son escépticos ante las posibilidades de éxito de la aplicación de recetas, sea en el exterior o el interior, que en circunstancias peculiares obtuvieron buenos resultados. No se sienten suficientemente seguros de que su historia haya sido un éxito como para desear extender sus lecciones al universo entero. Pero los norteamericanos, cuya historia es una historia exitosa, tienden a creer que los valores que surgen de su experiencia son de aplicación universal y dilícilmente admiten que éstos están atados a las condiciones especiales que permitieron que el éxito norteamericano fuese posible.

La larga experiencia de Estados Unidos llena de autoconfianza ha sido acompanada de complicaciones globales que, aunque a menudo brillantemente dirigidas, han acarreado enorme desencanto $y$ frustración. Lo último 
hubiese sido mucho menor si las expectativas engendradas por el feliz pasado estadounidense hubiesen sido más modestas; tal como son, perpetuaron una lánguida nostalgia por un pasado que dificilmente podria ser más distinto que el presente. Esto no ha paralizado la politica exterior norteamericana; ha creado sin embargo una tensión permanente entre las necesidades de la situación externa y las necesidades y esperanzas sicológicas más profundas de Estados Unidos y han conformado el talante y modo con que la nación recoge el guante en los desafios.

Una manifestación de esta desprendida visión norteamericana de la historia es su tendencia a descontar el peso del pasado. Este acto de fe intelectual en la "modernidad" no es meramente el distintivo de una sociedad 'lockeana' educada en el liberalismo, porque el liberalismo y la fe en el progreso no son mellizos siameses. Es éste el signo de una sociedad que lee su propia historia como una especie de largo prólogo a su presente y futuro; cuyo prolongado aislamiento ha significado que la historia de otros haya sido aprendida más bien que experimentada; mientras que en Europa la historia de cada nación es $\tan$ ponderosa, $\tan$ intimamente entrelazada con la historia de otras naciones, que la herencia de monumentos y recuerdos está profundamente embebida en la conciencia de todos. Es tambien el signo de una sociedad cuya fe en el progreso asume formas desconocidas en otros lugares, con miras no tanto hacia gradual (o cataclismico) mejoramiento y transformación de la historia - malos padres engendrando hijos mejores, quienes llegado su turno. engendrarán hijos aun mejores, etc. - sino hacia un abandono de la historia, una virginidad histórica perpetuamente renovada. Contrastando con su dificultad sicológica para llegar a un acuerdo con los habitantes de América Latina - descendientes de europeos que habian sido rechazados por Estados Unidos y de indigenas a quienes en su propio pais los norteamericanos destruyeron- el entusiasmo demostrado por muchos estudiantes norteamericanos por "conocer Africa" muy probablemente puede ser causado por algún tipo de afinidad sentida entre una tabula rasa y otra.

La amenazada satisfacción norteamericana asume otros variados disfraces. Uno de ellos, discutido a menudo por los escritores sobre politica exterior de Estados Unidos, es la presunción de una armonia final estable, una feliz mezcla de ingredientes liberales y conservadores. No concuerdan con esto los estadistas que miran las relaciones internacionales como un permanente test de voluntades. Innumerables proposiciones hacia el control de armamentos o medidas de política mundial han tenido en vista esta meta 0 concepción de estabilidad internacional. En realidad, el aséptico concepto de un mundo limpio de politicas de poder, o en el que los instrumentos de poder están lo suficientemente desafilados como para que las conductas 
Stanley Hoffmann / El estilo norteamericano: su pasado, sus principios

politicas internacionales desemboquen sólo en querellas marginales, enfrentamientos simbólicos o crecientes compromisos, puede encontrarse en los más sorprendentes contextos, tales como las sofisticadas discusiones sobre el control de armamentos en los años recientes. La historia es una llanura en la cual los pioneros avanzan, no una cadena montañosa, sin cumbres ni valles a la vista. La politica exterior se mira no como una permanente interacción de fuerzas caleidoscópicas e individuales, un continuum de conflictos y de crisis, sino como una actividad diseñada para disuadir y evitar daños ocasionales que podrian aminorar la velocidad en la marcha; como una serie de operaciones de rescate diseñadas para recuperar al viajero estancado y traerle nuevamente a la llanura; el evitar problemas y el manejar crisis definen la tarea; es una especie de solucionismo que se niega a morir.

Obviamente, sin embargo, esta complacencia está obstaculizada por la intratabilidad de los problemas en cuestión. Y siendo inevitable que los sucesos no satisfazgan las expectativas, los norteamericanos tienden a culpar al mundo (vale decir, a los otros) antes que a sus propias expectativas. En especial, un pueblo que jamás ha experimentado la derrota, siempre estará dispuesto a considerar la mera posibilidad de derrotas, hasta las más locales, como una catástrofe, muchisimo más que los pueblos que están acostumbrados a las alternativas del éxito y el fracaso y cuyas expectativas son más melancólicamente modestas.

Este distanciamiento de la historia y esta satısfacción con respecto de la misma convergen en la frecuentemente expresada convicción de que estamos por parte de la historia, o viceversa, y que nuestros enemigos no lo están. Otras naciones también tienden a creer que ellos son los predilectos de la historia. En el caso de Norteamérica, esta convicción es notable por la contradicción existente entre el determinismo implicito -aparentamos saber qué es lo que la historia condenará al cementerio y qué es lo que ella elegirá para el futuro-y la ironia o ira que un determinismo comparable provoca cuando es expresado por opositores marxistas. Y sin embargo, siendo que las convicciones marxistas descansan sobre un análisis (por muy desequilibrado y tosco que sea) de los procesos económicos y sociales y que una creencia en ellos podria considerarse un acto de fe empiricamente fundamentado, en las circunstancias norteamericanas las bases empiricas pertenecen generalmente al tipo de pensamientos benévolos y su acto de fe es una especie de susurro en la oscuridad.

Otra paradoja de la experiencia histórica norteamericana que afecta la relación de Estados Unidos con otras naciones es la dificultad para cooperar con ellas como iguales. La sociedad norteamericana nacida libre de las desigualdades del feudalismo y de la jerarquizada conciencia de clases de la sociedad industrial postfeudal, con el resto del mundo no ha sido igualizante. En este sentido, su historia es virtualmente opuesta a la de los paises más 
importantes de Europa, mucho menos igualitarios domésticamente pero que por necesidad lo son muchísimo más dentro de la sociedad europea.

Tenemos aqui tres consecuencias o efectos de la herencia norteamericana. En primer lugar, considerando que todas las antiguas grandes potencias (incluyendo a la Unión Soviética) han sido grandes potencias durante periodos en el transcurso de los cuales no existian ni amistades ni enemistades preestablecidas (aun el antagonismo franco-germano no fue hereditario antes de 1870), el compromiso de Estados Unidos en los asuntos mundiales ha ocurrido siempre durante periodos revolucionarios de hostilidad absoluta, aunque ciertamente el enemigo no ha sido siempre el mismo. En lugar de una sucesión de antagonismos limitados y de cooperaciones limitadas, caracteristica de los periodos moderados, Estados Unidos ha experimentado una sucesión de enemistades ilimitadas. Alemania se transformó en un aliado después de haber sido un odiado adversario, no porque la contienda mundial se hubiese enfriado sino porque una guerra fria total contra un nuevo adversario reemplazó a la verdadera guerra total. A medida que disminuye el antagonismo hacia la Unión Soviética, se le sustituye por un antagonismo hacia China Roja. En otras palabras, el potencialmente igual era el antiguo enemigo, no el aliado.

Segundo, aquellos hacia quienes Estados Unidos ha demostrado amistad no han sido sus iguales: han sido dependientes, abarcando desde América Latina a partir del siglo xvil hasta Gran Bretaña durante la Segunda Guerra Mundial —distintos tipos de dependientes, para decir verdad, desule subordinados hasta clientes, pero siempre suficientemente en segundo plano como para privar a Estados Unidos de la experiencia de una coalición de iguales. La única vez que Estados Unidos perteneció a una coalición de ese tipo, en 1917-19, la experiencia pareció ser insoportable. Finalmente prevaleció el aislacionismo, Norteamérica volvió a casa.

Tercero, la larga tradición de ausencia de complicaciones con los asuntos extranjeros otorgó a la diplomacia norteamericana una cierta limitación rigida, que el súbito vuelco hasta el extremo de compromiso total en una contienda ilimitada no logró superar completamente. La tradición de Estados Unidos era la de una acción unilateral (principalmente por la fuerza) y de una diplomacia altamente tradicionalista - majestuosa representación y observación en los más altos niveles. Es significativa la resistencia demostrada por tantos de los funcionarios más antiguos del Servicio Exterior (los que ya han alcanzado edad como para escribir sus memorias) hacia las innovaciones requeridas por la diplomacia total de la década actual-propaganda, ayuda económica, Cuerpo de Paz, relaciones públicas, etc. No fue para esto que fueron adiestrados, pero es precisamente esto lo que entraña una politica de compromiso internacional cooperativo hoy en dia.

Puede ser sumamente irritante tener que cooperar con prójimos cuya 
Stanley Hoffmann / El estilo norteamericano: su pasado, sus principios

relativa inmunidad o vigorosa resistencia a la presión puede darles una especie de igualdad compensatoria, a pesar del propio tamaño y predominio. Pero para otros (especialmente en Europa) la interdependencia es la historia más antigua del mundo. Han vivido siempre amontonados cual sardinas: en una lata y están demasiado acostumbrados a ver que de vez en cuando uno de ellos se las da de tiburón. Para los norteamericanos, la interdependencia significa una especie de declinación de su soberania y una contribución hacia la superación de la nación-estado. Para otros estados, la interdependencia es la norma y no afecta seriamente la esencia de su soberania: lo que modifica la soberania es la dependencia (como Rousseau tan bien lo comprendia), tener que estar sujeto a las exigencias y órdenes de otros. Para nosotros, la soberanía parece roerse y disminuirse por el compromiso. La soberania se pierde no por medio de medidas cooperativas sino únicamente por transferencias de poder.

El pasado norteamericano ha procreado expectativas y conformado percepciones que podrian ser perjudiciales para la libre elección y para la acción efectiva dentro de los asuntos extranjeros. Pero por lo mismo que la historia de Norteamérica ha sido la de un crisol nacional y porque ha sido tan afortunada con el éxito, también ha procreado una serie de creencias que en conjunto sirven para acentuar los mitos históricos; y por otra parte sirven también en la función o disfunción de proveer normas para la evaluación y la acción.

Estados Unidos no es una nación ideológica ni su politica es ideológica, si por ideologia comprendemos un cuerpo de ideas, emociones y simbolos que aspiran a presentar una visión sistemática y global del universo y su historia, que sirven para comprometerse en, guiarse hacia, y como legitimización de la acción, que se institucionalizan dentro de un movimiento politico organizado. Una ideologia en este sentido es intensamente operacional por sus correas de transmisión institucionales, su dinamismo y lo que yo denominaria su outer-directedness ${ }^{1}$, presumiblemente colocando en manos de sus paladines la llave o el juego de llaves que servirán para abrir todas las puertas, una palanca con la cual mover hombres y montañas. Más aún, provee de categorias para la ordenación de datos, explicaciones para los sucesos y comportamientos $y$, de vez en cuando, proyecciones para iluminar el futuro.

Por otro lado, los principios norteamericanos no proporcionan el tipo de lineas guias acción no-ideológica o tradiciones que algunas naciones

'Outer directedness, dirección hacin afuera; inner-directedness, dirección hacia adentro; definiciones de D. Riesman, Harvard University, utilizadas comúnmente en inglés. (Nota del traductor). 
mantienen aún en su politica exterior - reglas prácticas que definen ciertos objetivos, tales como la preservación del equilibrio de poderes, en el caso británico, o la llamada tradición de fronteras naturales o de l'alliance de revers en el francés. Lo que distingue a los principios norteamericanos en asuntos extranjeros de dichas lineas-guias es en realidad algo que aquéllas comparten con la ideologia.

Las lineas-guias son por lo general conservadoras y con esto quiero decir, ya sea: a) diseñadas para mantener la totalidad del statu quo en concordancia con los intereses de la nación, o b) diseñadas para formentar ambiciones revisionistas dentro de un sistema de relaciones internacionales que se cree poseedor de un molde permanente o necesario. Las ideologias, en la medida que se ocupan de asuntos internacionales, son tipicamente revolucionarias -no importando si la revolución consiste en adelantar o retrasar las manecillas del reloj. El sistema mismo debe ser hecho cenizas y es la nación ideológica la que debe Ilevar la antorcha.

Los principios de Estados Unidos comparten dos rasgos con las ideologias: uno negativo, la supuesta trascendencia respecto de sus intereses nacionales (vale decir, éstos no estarian diseñados primariamente para promover particularmente las ambiciones o intereses norteamericanos, aunque de su éxito obtenga ese resultado); el otro, positivo, en que en los asuntos externos estos principios van más allá del estrecho universo de maniobras interestatales y expresen opiniones generalizadas del hombre y la sociedad.

Sin embargo estos principios difieren profundamente de la ideologia. No se encuentran institucionalizados: no existe algún partido, o maquinaria estatal comprometida en llevar la antorcha y quemar a los no creyentes. No tienen ra cohesión e interrelación de los dogmas ideológicos y normalmente no inspiran el mismo grado de fervor, aunque seria en verdad imprudente generalizar sobre este punto. Más importante aún, la visión que expresan es reformista en lugar de revolucionaria y no se encuentran tan históricamente fundamentados como las ideologias. Si bien Estados Unidos espera adaptar su vida politica dentro de ciertas lineas, y contempla un cambio global, su meta es un progreso sin apocalipsis. La visión norteamericana no posee las profecias y apreciadas revelaciones de las ideologias y. se contenta con embellecimientos y mejoramientos sobre un diseño básicamente aceptado, en lugar del repudio total de este diseño. Las ideologias están históricamente enraizadas - con lo cual no quiero decir que éstas interpreten correctamente las tendencias históricas ni proporcionen instrumentos a prueba de chambones para la acción, sino que simplemente ellas proveen tanto explicaciones históricas como objetivos históricos, medios de acción y combustible para la fe.

Es cierto que los principios de Norteamérica son, en dos sentidos, profun- 
Stanley Hoffmann / El estilo nòrteamericano: su pasado, sus principios

damente históricos. Por un lado, "los idolos que Norteamérica venera son. . los idolos de su propia cultura traspuestos al escenario mundial", frutos de las primeras creencias y experiencia histórica más temprana. Pero el inconveniente de esto seria precisamente su inner-directedness, porque, como sabemos, el escenario norteamericano y el teatro mundial difieren profundamente. Por otro lado, algunos de sus principios si que reflejan la más reciente experiencia en politica exterior de Estados Unidos. Aqui el inconveniente seria que lo que debia ser una linea flexible y condicional se torna en un dogma que pierde contacto con la realidad histórica y sus complejidades.

Los principios norteamericanos caben en dos categorias: primero, dogmas abstractos e imperativos morales, profundamente sentidos y ampliamente compartidos, que establecen metas y definen reglas de conducta; segundo, supuestos sobre conductas que pretenden proporcionar métodos de acción para lograr las metas establecidas. Estos imperativos y postulados explican la peculiar ambigüedad de la politica exterior de Estados Unidos.

Son una fuente de fortaleza, precisamente porque expresan creencias y experiencias intensamente estimadas, porque están tan próximos a la esencia norteamericana. Otorgan a Estados Unidos su fuerza evangelizadora, su tono misionero. Es cierto que durante largo tiempo se interpretó que la noción de ser un pueblo elegido requeria una separación con el mundo, pero en cuanto esta separación se hizo imposible, entonces se consideró que la acción en la arena internacional se justificaba siempre que su fin fuese la reforma, por medio del fomento de estos principios. A menudo oimos mencionar la idea francesa sobre una mission civilisatrice (como una meta de política externa se extendió sólo a las colonias de Francia); en el caso norteamericano, tendriamos que hablar de una misión emancipadora - primeramente del mundo externo, en seguida de este mundo. La transposición deliberada de politicas a seguir, decidida sobre las bases de poderio o del interés nacional, hacia el lenguaje de los principios, corresponde asi a dos supuestos caracteristicos: que éste es el lenguaje que mejor conmueve al pueblo norteamericano porque es el suyo propio; y que éste es el lenguaje con mayores probabilidades de eficacia en el exterior. El primer postulado refleja una experiencia, el segundo únicamente una convicción.

Pero estos principios son también una grave fuente de debilidad en los asuntos internacionales. Son como botellas lanzadas a las olas, luces de Bengala en la noche, llamados a través de la neblina, que uno nunca sabe si serán reconocidos o escuchados. A veces si lo son, porque resulta que calzan en una situación dada. Entonces el éxito estimula a Estados Unidos para probarlo una y otra vez. Sorpresa y desaliento son las reacciones cuando la batalla se pierde, cuando las luces no iluminan paisaje alguno, cuando al

2. Iiax Lerner, America as a Civilization. Nueva York: Simon and Schuster, 1957, p. 220. 
EST T D I O S I $\therefore$ T E R N A C I O N A L E S

llamado contesta sólo un eco nuestro. Porque los principios de Norteamérica son desencarnados. Son como un vino que intoxica al viñatero pero que el resto de los catadores encuentra con poco cuerpo. Aunque poseen un elemento a priori considerable, no obstante sus normas están fundamentadas en su mayor medida en las realidades politicas y sociales de Estados Unidos.

Ahora, tanto las ideologias como las lineas de acción indican metas concretas, revelan conexiones, señalan sectores pertinentes para la acción, muestran enemigos concretos. Esto podria explicar por qué el revisionista y el revolucionario son tan a menudo aliados, por qué el conservador del statu quo y el revolucionario pueden estar de acuerdo durante una tregua ocasional, negociada por vias opuestas pero con el mismo lenguaje de historia politica. La visión reformista, por otro lado, que a menudo comparte elevados fines universales con la revolucionaria, no se fundamenta suficientemente en la historia como para ser totalmente operacional, ni es bastante flexible o maquiavélica como para proporcionar un tipo de dirección a las relaciones de poder con el cual los conservadores del statu quo o los revisionistas sobresalgan. Tipicamente, esta visión consiste en fines sin medios - listas de compras con los precios sin marcar-o en hipersimplificadas nociones sobre los medios. La Declaración de Independencia no es una Carta que baste para realizar politica. El Manifiesto Comunista y los distintos programas del Partido Comunista de la Unión Soviética comparten su propia parte de hipersimplificación, distorsión e ilusiones; pero también contienen una cantidad suficiente de método analitico, una suficiente captación del eslabonamiento social, una suficiente cantidad de enfoques sobre las tendencias históricas como para ser capaz de corregir sus propios errores en un grado considerable.

Estados Unidos escapó del bizantinismo ideológico, pero no logró evitar una dañina oscilación entre principios, postulados poco practicables, y análisis sociológicos sofisticados pero también inconcluyentes, que son aún menos adecuados como guias para la acción que los principios o las ideologias. Ni tampoco ha podido evitar una de las consecuencias más paradojales de la descarnada naturaleza de los principios: el hecho de que éstos ni mueren ni se borran (una ideologia puede ser erosionada por la rutinización de su apoyo institucional, corroida por repetidos errores de interpretación y fracasos en la acción). Los dogmas y principios ideológicos descansan sobre diferentes tipos de fe: una es entusiasmante, demónica, dinámica, activista; la otra es simplemente esperanza. La primera es altamente explosiva, sin embargo no sobrevive al fracaso continuado o repetido $y$ se torna entonces en cinismo o indiferencia pasiva y amarga. Pero la esperanza mana eternamente. . . 
Sólo me es posible aqui citar unos pocos ejemplos de los tipos de principios a que me refiero. Los norteamericanos no son los únicos que creen en ellos; pero están solos en su determinación de actuar en acuerdo con estos principios, como si la única lección derivada de tristes experiencias fuese la que el caballo de Orwell, en Animal Farm, extraia de sus fracasos: "Trabajaré más" y lo intentaré con mayor ahinco.

Dentro de la categoria de los imperativos morales, dos merecen una breve discusión: el principio de autodeterminación y el principio según el cual ningún cambio sobre el statu quo puede perpetrarse por medio de la fuerza (llamémoslo el principio de cambio pacifico). Ambos corresponden tanto a experiencias domésticas norteamericanas proyectadas hacia los asuntos exteriores como a experiencias de politica externa momificadas.

La critica a la noción de autodeterminación es tan antigua como los Catorce Puntos de Wilson, y no existe necesidad alguna de repetir los argumentos tan a menudo invocados sobre la indeterminación de la autodeterminación, el problema de las minorias nacionales, la contradicción entre los derechos individuales y colectivos. Lo que es pertinente aqui, en nuestra discusión, es que el principio mismo representa una admirable mezcla de ideales y egoismo - el ideal de la libertad compartido por la gran parte del mundo occidental y el propio interés de utilizar como un fin y un acicate para otros una noción politica que corresponde al sentido de legitimidad prevaleciente, y que contradice la confianza de los adversarios en la acción violenta ejecutada por grupos minoritarios, su preferencia por la coerción sobre el consenso, o su maquinación de algún tipo de rudimentario consenso. No obstante, aunque fuese siempre clara la naturaleza de la unidad en la cual ha de concederse la autodeterminación, o aunque el problema no fuese un problema de nacionalismo sino de régimen, aun asi restarian dos dolores de cabeza que la dedicación de Estados Unidos a este principio despierta.

Primero, está la dudosa presuncion de que la aplicación universal del principio de autodeterminación siempre resultará bien -que ninguna nación jamás llegará al comunismo por elecciones, como la gastada frase indica. Esta presunción, es un supuesto que a su turno se basa en los postulados de que ninguna nación por su propia voluntad buscará una dictadura y que para personas resueltas en la búsqueda de la independencia nacional el comunismo en todas partes y obviamente siempre significará subordinación a Moscú o Pekin. En la medida en que todos estos postulados pueden ponerse en tela de juicio, un apoyo incondicional de la autodeterminación arriesga un peligroso conflicto entre el ideal y el propio interés. Con toda seguridad uno podria argumentar que en vista de que los postulados son correctos, a la larga y en la mayoria de los casos también a la corta, una mejor definición de los intereses norteamericanos permitiria la aceptación del principio aun cuando y donde su aplicación vaya en contra de 
nuestro interés inmediato. Pero la incógnita de si es éste un buen argumento deberá ser resuelta caso por caso y no sobre la base del solo principio.

Esto nos lleva al segundo quebradero de cabeza, que lo produce el hecho de que el principio de autodeterminación únicamente puede funcionar a través de maquinarias e instituciones; la pregunta clave es a menudo no tanto si un pueblo será capaz de determinar cuál será su gobierno, sino más bien si contará èste con las alternativas e instrumentos politicos eleccionarios sin los cuales un voto seria una farsa. Porque la autodeterminación, como la mayoria de los valores y deidades, es algo que debe ser mediado. "El pueblo" sólo ocasionalmente es una fuerza de energia; casi siempre es una fuerza de reserva o un árbitro final. Cuando no existen potenciales triunfadores organizados, cuando el pueblo es una masa incoada, o cuando hay solamente una fuerza organizada, el problema de la autodeterminación es discutible.

El principio del cambio pacifico (que lleva como su colorario el que Estados Unidos debe apoyar o fomentar la resistencia a la agresión) presenta algunos de los mismos beneficios y problemas. Una vez más se mezclan los propios intereses y los ideales. Una vez más, las mayores molestias surgen de su aplicación. Una vez más puede que se destruya a si mismo o que simplemente sea no operativo. Le destruirá a si mismo toda vez que los Estados Unidos enfrente una situación donde sus intereses aparenten dictar la necesidad de cruzar una frontera con fuerzas o por la fuerza, y sin la invitación del gobierno en el poder. El argumento de que una sólida definición de cuál seria el interés norteamericano eliminaria cualquier violación del principio es únicamente una petición de principios. Porque la competencia a menudo dicta sus propios reglamentos y puede que exista una abrumadora necesidad de violar el principio, ya sea por razones de posesión o de milieu, sea para salvaguardar intereses concretos o para defender un valor simbólico tal como el prestigio. Con toda seguridad un casuista podria argumentar que una medida como ésta no seria agresiva cuando el propósito es derrocar a un régimen comunista, que en si mismo ha sido establecido por la fuerza, o cuando es para prevenir una toma de posesión comunista ejecutada por la fuerza. ¿Pero entonces el régimen asi establecido es en algo menos coercitivo porque fue introducido por el paladín del mundo libre? $¿$ Puede equipararse un régimen establecido por libres elecciones o por una revolución popular, que el curso de los acontecimientos arrastra después hacia el comunismo, con un régimen impuesto por la fuerza?

O sencillamente el principio de cambio pacifico carezca quizá de importancia. Porque, tal como lo descubrieron los especialistas que desperdiciaron su ingenio y astucia intentando definir la agresión, la noción de agresión es únicamente aplicable cuando existe una violación de limites claramente definida, realizada por un ejército extranjero, de la frontera de un estado bien establecido con un gobierno ordenadamente a cargo del mismo. La misma idea de agresión corresponde a una nitida dicotomia 
pacifica en las relaciones internacionales y a una situación de estabilidad doméstica. Su aplicabilidad durante una era de ni paz ni guerra es altamente dudosa - de ahi el colapso de tantas de las reglas de la legislación internacional que tratan sobre las guerras civiles o interestatales. En el caso de conflictos prolongados, donde es casi imposible descubrir quién asestó el primer golpe, y más aún en el caso de guerras civiles entabladas alrededor de regimenes vacilantes, el principio de resistencia a la agresión tiende a transformarse en una racionalización invocada muy fácilmente por todas las partes. Y la noción de cambio pacífico en algunos casos frecuentemente está en conflicto con la ausencia de cualquier tipo de canal para el pacifico resarcimiento de quejas o el arreglo de disputas; en otros está en conflicto con el principio que los norteamericanos comúnmente veneran, el derecho a la revolución. La conciliación de ese derecho con el dogma de cambio pacifico es una tarea formidable.

Ciertos principios norteamericanos caben dentro de la categoria de los supuestos (al igual que los dogmas, descansan sobre supuestos implicitos y contienen metas implicitas). Podemos decir unas pocas palabras sobre dos de ellos. El primero es uno que penetra todos los planes y programas norteamericanos para las regiones subdesarrolladas; es el supuesto de que el principal problema que surge en las naciones recientemente independizadas es el desarrollo económico. Se presupone, primero, que el comportamiento de la nueva nación estará determinado en gran medida por el nivel de su deșarrollo económico; segundo, que el régimen doméstico es en medida considerable una función del nivel de desarrollo; tercero, que, si es correcta, la acción del sector económico de la sociedad logrará producir resultados beneficiosos en los otros sectores; cuarto, que la meta correcta de un lider genuino en una de estas naciones debiera ser el desarrollo de su pais. Y la meta implicita en esta serie de postulados es que Estados Unidos debiera concentrarse en ayudar a las economias subdesarrolladas a alcanzar la etapa de crecimiento autosustentado. No es una mala meta, pero si los postulados resultan sólo en parte ciertos, la concentración en ella no necesariamente conducirá a los resultados que se esperaron, y podrán darse instancias en las que deberá ser abandonada.

Es obvio que el desarrollo económico es una necesidad urgente, y que aquellos lideres politicos que lo descuiden no mandarán por mucho tiempo. Pero, en el mundo de hoy son muy raros los lideres politicos que consideran como un fin supremo al desarrollo económico, o como una meta desconectada de muchas otras; algunas de ellas probablemente hasta podrán aminorar el desarrollo, y todas ellas mostrarán la tendencia a empapar esta meta abstracta con el color y el sabor de lo politico. La experiencia de las naciones recién nacidas no difiere en mucho de la experiencia de las naciones del mundo occidental durante una etapa comparable: sus lideres están impulsados por un formidable conjunto de motivaciones, entre las cuales 
(por las razones ya mencionadas) el deseo de brillar en el tablado mundial, el impulso hacia la consolidación del control doméstico, la determinación de integrar elementos dispares de la población o de lograr ciertas reformas sociales figuran con tanta prominencia como el desarrollo.

Esto significa que el desarrollo económico es a menudo considerado como un medio para otros fines. Consiguientemente, la ayuda al desarrollo será a menudo apreciada sólo en la medida en que ella promueva otros fines; o el desarrollo quedará minimizado si, dentro de la jerarquia de fines del lider, aquellos objetivos para los cuales el crecimiento económico es un requisito previo están colocados muy abajo o si aquéllos colocados más alto pueden peligrar con la aceptación de la ayuda externa. Hay mucho de verdad en el argumento de que la ausencia del desarrollo significa asegurarse el caos (el contrargumento de que el desarrollo muchas veces lleva a una dislocación y disturbios, aunque ocasionalmente veraz, no acaba con el caso). Pero puede suceder que el desarrollo lleve a una distinta especie de irresponsabilidad - la de una sociedad complacientemente inward-looking que saborea su creciente prosperidad y deja para otros las molestias.

Tras este supuesto sobre el desarrollo económico encontramos entonces, más que un análisis de la escena mundial, una proyección idealizada de la propia experiencia norteamericana de crecimiento económico, que se dio acompañada de una gran cantidad de estabilidad social, progreso social y democracia politica; la proyección de una nación donde por mucho tiempo los estadistas se "redujeron a efectuar progresos en casa en lugar de molestar en el exterior"3 y sin embargo no sintieron remordimiento alguno de estar mirando hacia adentro, porque pensaban que el mundo exterior era bastante feo. ¿Pero cómo podriamos olvidar que las riquezas y los componentes sociales de Estados Unidos fueron casi únicos; que sus instituciones precedieron y posteriormente conformaron y canalizaron el crecimiento económico norteamericano (como lo demuestra la persistente validez del análisis de Tocqueville, escrito antes de la época del consumo en masa); que la abundancia de Estados Unidos, el consenso por una sociedad liberal y la limitación voluntaria de objetivos en el exterior eximió a Estados Unidos de esa dolorosa alternativa entre igualmente deseables pero no simultáneamente alcanzables fines, que confrontan a las sociedades menos ricas en bienes, pero más acaudaladas en metas conflictivas; y que la concentración en problemas domésticos sin lugar a dudas trajo consigo turbulencia y algo de negligencia irresponsable en el exterior, y que en todo caso fue permitida por un sistema internacional completamente diferente? Porque para Estados Unidos también el desarrollo económico fue un trampolin, un medio de acción, un requisito previo al poderio mundial; pero debido a su prolongado aislamiento y su aversión hacia ese poderio, los norteamericanos oscilaron

Arthur Schlesinger, A Thousand Days, Nueva York: Houghton Mifflin, 1965, p. 567 
entre la opinión de que respecto del pais los negocios son negocios y la opinión de que su negocio era la emancipación mundial - dos creencias que tienden a aislar al proceso de desarrollo económico del molde de los asuntos exteriores.

Otro supuesto frecuente (o conjunto de supuestos) se resume con la palabra consenso. Los norteamericanos están convencidos de lo que en otra parte he llamado la ilusión del procedimiento: que el consenso no solamente es posible, como el resultado de adaptaciones mutuas entre hombres de buena fe, sino que es la base sobre la cual los lideres pueden elegir sus alternativas. El fin implicito es, por supuesto, alcanzar el consenso. Lo que uno descubre detrás de esta noción es una cierta concepción de la racionalidad - una fe en la existencia de un único tipo de racionalidad, a menudo confundida por prejuicios y malas costumbres, pero, tal como la voluntad general de Rousseau, dispuesta a brillar en el momento mismo en que las capas de suciedad y polvo sean retiradas. Uno también encuentra el concepto hermano de una armonía última: igual que existe una sola racionalidad entre parcialidades en competencia, existe una comunidad en potencia tras los intereses en conflicto. Dado que la diversidad de opiniones no impide el triunfo de la razón y de la armonia, es bastante cierto que la comunidad y la diversidad son gemelas. Tercero, uno encuentra implicitamente asumido el universo del utilitarianismo, el que define a los valores en términos mensurables y aspira al mayor bien para los más numerosos.

Estas nociones nos llevan hacia una definición de la politica como relaciones interpersonales. Existe una visión implícita de un continuum de pequeños grupos y politicas internacionales; cada conjunto mayor parece ser reducible $y$ susceptible de análisis en términos de un pequeño grupo de demandantes buscando a tientas el consenso por medio de la que se ha llamado adaptación mutua parcial.

Lo que para este autor resulta ser un pecado de trasposición — desde un tipo de acción social a una muy distinta forma de realidad-, es, una vez más, una proyección de la experiencia norteamericana; este seria el ethos liberal escrito con grandes mayúsculas; la sociedad liberal superimpuesta sobre un estado de la naturaleza. Porque en la trastienda de todo esto, existe una especie de supermodelo de una comunidad cuyos miembros comparten creencias fundamentales (incluyéndose esta fe incondicional en un cierto procedimiento de adaptación). Se regatea por un poco más o un poco menos en la distribución de los bienes, pero hay consenso en la necesidad de acordar, y la adaptación está facilitada por un sistema de reglamentos y convenciones. En una comunidad parecida, está en juego, en realidad sólo lo que Charles Lindblom ha llamado "alternativas marginales relativamente concretas", donde el intercambio se favorece con la convicción de que mi pérdida de hoy dia quedará compensada por mi ganancia de mañana. El único punto en común con el estado de la naturaleza internacional es la 
E S T U D I O S I N T E R N A C I O N A L E S

multiplicidad de demandantes. Pero los demandantes nacionales no están bajo ninguna compulsión mental o física para definir valores de cierta manera, para regatear sólo en los márgenes, o de llegar a un acuerdo. En un caso la falta de un poder dominante es un incentivo para la adaptación mutua, en el otro es una desventaja. La concepción de la democracia no es la de las relaciones internacionales.

Lo que Agustin dijo sobre la búsqueda de Dios se aplica a la búsqueda del consenso: "Vosotros no me buscariais si ya no me hubieseis encontrado". El consenso es posible cuando desde la partida existe base suficiente para una comunidad. Cuando existe una armonia fundamental sobre los fines y se está . en desacuerdo únicamente sobre los medios, o cuando los medios son tan abundantes como para conciliar distintas jerarquias de fines, el pluralismo es eficaz y creativo. El consenso último emana del consenso en los procedimientos que forjó el debate y que no seria extraño fuese suficientemente claro como para incitar y guiar a la acción. Cuando no existe una armonia preestablecida dentro de un universo de unidades rivalizantes, cada una dueña de su propia racionalidad, se requiere más que buena voluntad y relaciones personales para establecer el acuerdo; la alternativa reside entre un consenso verbal que lleva hasta el máximo la ambigüedad, paralizando la política, y la discordia. Estados Unidos, especialmente en su versión autoidealizada, es en grado sumo una sociedad rousseauniana, accionada por una visión de la voluntad general y marcada por una fuerte presión social hacia el conformismo. Pero el consumado pesimista que fue Rousseau jamás aplicó el contrato social a la escena internacional. 